opportunities to test high risk groups such as MSM, who are less likely than women to be HIV tested in this setting. Interventions are urgently needed to increase HIV testing among STD patients.

\section{5-S4.04 TARGETED STD SCREENING AMONG HIGH-RISK MEN WHO HAVE SEX WITH MEN}

doi:10.1136/sextrans-2011-050109.172

R Perkins, B Furness, M Hager, R McLaughlin, J Goforth, M Davies. Whitman-Walker Clinic, Washington, USA

Background Subgroups of men who have sex with men (MSM) are at high risk for HIV infection and other viral and bacterial STDs. Since the mid-1990s, increased rates of early syphilis, gonorrhoea, and chlamydial infection and higher rates of unsafe sexual behaviours have been documented among MSM in the USA and virtually all industrialised countries.

Methods The Gay Men's Health \& Wellness (GMH\&W) Clinic is a Tuesday and Thursday evening STD clinic for men at WhitmanWalker Clinic (WWC), a Washington, DC community health center specialising in HIV/AIDS care and lesbian, gay, bisexual, and transgender care. As an outreach of this bi-weekly clinic, WWC, Gilead Foundation, and the local HIV/AIDS, Hepatitis, STD, and TB Administration collaborated to provide STD screening in the Crew Club, the District's only bathhouse serving MSM, for $\sim 4 \mathrm{~h}$ each Tuesday night beginning July 2010. All participating men were offered HIV screening, syphilis screening, and nucleic acid amplification testing NAAT) for gonorrhoea and chlamydia of urine, pharyngeal, and rectal specimens.

Results During the first 6 months of testing at the Crew Club, 173 unique clients were screened. Of those, five new HIV and 18 new syphilis cases were identified (Abstract O5-S4.04 table 1). In addition, two urethral, six pharyngeal, and two rectal gonorrhoeal infections were identified and five urethral, two pharyngeal, and 14 rectal chlamydial infections were identified. In comparison, during 2010, 2487 unique clients were tested at the GMH\&W Clinic. Of those, $37 \mathrm{HIV}$ and 189 syphilis cases were identified. In addition, 115 urethral and 89 rectal gonorrhoeal infections were identified and 139 urethral and 124 rectal chlamydial infections were identified. Pharyngeal results from the GMH\&W Clinic were not yet available.

Abstract 05-S4.04 Table 1 Testing results, gay men's health \& wellness clinic (2010) vs STD Screening at the Crew Club (July-December 2010)

\begin{tabular}{llllllll}
\hline & \multicolumn{3}{l}{ GMH\&W clinic } & \multicolumn{3}{l}{ Crew club } \\
\cline { 2 - 3 } Test & Tests & Positives & \% Positive & & Tests & Positives & \% Positive \\
\hline HIV & 2747 & 37 & $1.35 \%$ & & 189 & 7 & $3.70 \%$ \\
Syphilis & 2969 & 189 & $6.37 \%$ & & 191 & 18 & $9.42 \%$ \\
Urethral GC & 1753 & 115 & $6.56 \%$ & & 184 & 2 & $1.09 \%$ \\
Pharyngeal GC & 2017 & N/A & - & & 189 & 6 & $3.17 \%$ \\
Rectal GC & 1138 & 89 & $7.82 \%$ & & 183 & 2 & $1.09 \%$ \\
Urethral CT & 1753 & 139 & $7.93 \%$ & & 184 & 5 & $2.72 \%$ \\
Pharyngeal CT & 2017 & N/A & - & & 189 & 2 & $1.06 \%$ \\
Rectal CT & 1138 & 124 & $10.90 \%$ & & 183 & 14 & $7.65 \%$ \\
\hline
\end{tabular}

Conclusions A higher percentage of presumably asymptomatic clients tested positive for HIV and syphilis infections during STD screening in this non-traditional venue than during the diagnosis and routine screening that occurred at the clinic. Conversely, a higher percentage of clients, many of whom were symptomatic, tested positive for gonorrhoea and chlamydia during the GMH\&W Clinic than during the Tuesday night outreach. This effort allows us to engage hard-to-reach men who may be among the core transmitters driving the HIV and syphilis epidemics in the DC area. Costs per HIV and syphilis cases detected need to be calculated /estimated for this fruitful outreach endeavour.

\section{5-S4.05 INTRODUCTION OF A SEXUAL HEALTH PRACTICE NURSE INCREASES STI TESTING AMONG MSM IN GENERAL PRACTICE}

doi:10.1136/sextrans-2011-050109.173

${ }^{1} \mathrm{M}$ Y Chen, ${ }^{1} \mathrm{~A}$ F Snow, ${ }^{1} \mathrm{R}$ Cummings, ${ }^{2} \mathrm{~L}$ Owen, ${ }^{3} \mathrm{C}$ El-Hyak, ${ }^{3} \mathrm{M}$ E Hellard, ${ }^{4} \mathrm{~L}$ Vodstrcil, ${ }^{5} \mathrm{C}$ K Fairley, ${ }^{5} \mathrm{M}$ Y Chen. ${ }^{1}$ Melbourne Sexual Health Centre, Melbourne, Australia; ${ }^{2}$ Victorian AIDS Council, Gay Mens Health Centre, Australia; ${ }^{3}$ Burnet Institute, Australia; ${ }^{4}$ University of Melbourne, Australia; ${ }^{5}$ Melbourne Sexual Health Centre, University of Melbourne, Australia

Introduction Increased screening among MSM could improve STI control in this population; however published data on interventions that improve screening rates is limited. The aim of this study was to determine if introducing a sexual health practice nurse (SHPN) into a general practice clinic could increase HIV and STI testing among MSM who attend.

Methods In October 2008, Melbourne Sexual Health Centre introduced a SHPN into a Melbourne general practice with a high caseload of MSM. We undertook an observational study comparing the proportion and STI tests undertaken in the 9 months before (Period 1), and after the SHPN was introduced (Period 2). Consistent with Australian national STI testing guidelines for MSM, complete testing was defined as HIV and syphilis serology, urine test and anal swab for chlamydia, pharyngeal and rectal swabs for gonorrhoea, from the same man on the same date. MSM were stratified and analysed according to HIV status. The Qui-Square Test for Independence was used to compare the difference in proportions of tests. In the case of syphilis tests among HIV positive MSM the median number of tests was compared using the Mann-Whittney U Test. Results Among HIV negative MSM, the propotion of MSM tested, increased from Period 1 to Period 2 as follows; HIV from $57.8 \%$ to $66.2 \%$; syphilis from $59.9 \%$ to $76.6 \%$; urethral chlamydia from $67.7 \%$ to $75.8 \%$; pharyngeal gonorrhoea $62.5 \%$ to $69.9 \%$; and rectal gonorrhoea/chlamydia from $58.5 \%$ to $69.5 \%$ (all $\mathrm{p}<0.001$ ). The proportion of episodes of complete tesing, also increased from $41.1 \%$ to $51.9 \%(\mathrm{p}<0.001)$. Among HIV positive MSM, the proportion of MSM tested, increased from Period 1 to Period 2 as follows; urethral chlamydia from $66.5 \%$ to $80.2 \%$, pharyngeal gonorrhoea $58.7 \%$ to $77.2 \%$ and rectal gonorrhoea/chlamydia from $55.3 \%$ to $75.3 \%$, (all $\mathrm{p}<0.001$ ). Prior to Period 1 , the clinic had a policy of testing HIV positive MSM for syphilis with each three monthly routine HIV monitoring blood tests. Therefore, the median number of syphilis tests was the same in both study periods (median 2, range 0 to 6 , $\mathrm{p}=0.817)$. The proportion of episodes of complete tesing, also increased from $32.3 \%$ to $56.2 \%(\mathrm{p}<0.001)$.

Conclusion The introduction of a SHPN into general practice significantly increased HIV and STI testing among MSM. The magnitude of the effect of this intervention may be greater in MSM high case load general practices where the culture of STI testing is less well established.

\section{5-S4.06 IMPROVING HIV SCREENING AT AN URBAN STD CLINIC}

doi:10.1136/sextrans-2011-050109.174

${ }^{1} \mathrm{~B}$ W Furness, ${ }^{2} \mathrm{~J}$ Coursey, ${ }^{2} \mathrm{~T}$ Flemming, ${ }^{2} \mathrm{P}$ Hess, ${ }^{3} \mathrm{~S}$ Sankar, ${ }^{3} \mathrm{~N}$ Kamanu Elias. ${ }^{1} \mathrm{CDC} /$ NCHHSTP/DSTDP/ESB/FEU, Washington, USA; ${ }^{2}$ CDC/NCHHSTP/DSTDP, Washington, USA; ${ }^{3}$ Department of Health, Washington, District of Columbia, USA

Background At the end of 2006 (the most recent year that data are available), an estimated 1106400 persons in the USA were living with HIV infection, with 21\% undiagnosed. In September 2006, the Centers for Disease Control \& Prevention (CDC) released new 